\title{
A note on coloring line arrangements
}

\author{
Eyal Ackerman \\ Department of Mathematics, Physics, and Computer Science \\ University of Haifa at Oranim \\ Tivon 36006, Israel \\ ackerman@sci.haifa.ac.il \\ János Pach* \\ EPFL, Lausanne, Switzerland \\ and Alfréd Rényi Institute, Budapest, Hungary \\ pach@cims.nyu.edu \\ Rom Pinchasi ${ }^{\dagger}$ \\ Mathematics Department \\ Technion-Israel Institute of Technology \\ Haifa 32000, Israel \\ room@math.technion.ac.il

\section{Radoš Radoičić} \\ Department of Mathematics, Baruch College \\ City University of New York, New York, U.S.A. \\ rados.radoicic@baruch. cuny.edu

\section{Géza Tóth} \\ Alfréd Rényi Institute \\ Budapest, Hungary \\ geza@renyi.hu
}

Submitted: Aug 26, 2012; Accepted: Apr 26, 2014; Published: May 9, 2014

Mathematics Subject Classifications: 52C30

\begin{abstract}
We show that the lines of every arrangement of $n$ lines in the plane can be colored with $O(\sqrt{n / \log n})$ colors such that no face of the arrangement is monochromatic. This improves a bound of Bose et al. by a $\Theta(\sqrt{\log n})$ factor. Any further improvement on this bound would also improve the best known lower bound on the following problem of Erdős: estimate the maximum number of points in general position within a set of $n$ points containing no four collinear points.
\end{abstract}

Keywords: Arrangements of lines, chromatic number, sparse hypergraphs.

*Supported by NSF grant CCF-08-30272, by Hungarian Science Foundation EuroGIGA Grant OTKA NN 102029, and by Swiss National Science Foundation grants 200021-137574 and 200020-144531.

${ }^{\dagger}$ Supported by ISF grant (grant No. 1357/12).

${ }^{\ddagger}$ Supported by Hungarian Science Foundation Grant OTKA K 83767 and NN 102029 . 


\section{Introduction}

Given a simple arrangement $\mathcal{A}$ of a set $L$ of lines in $\mathbb{R}^{2}$ (no parallel lines and no three lines going through the same point), decomposing the plane into the set $C$ of cells (i.e. maximal connected components of $\left.\mathbb{R}^{2} \backslash L\right)$, Bose et al. [1] defined a hypergraph $\mathcal{H}_{\text {line-cell }}=(L, C)$ with the vertex set $L$ (the set of lines of $\mathcal{A}$ ), and each hyperedge $c \in C$ being defined by the set of lines forming the boundary of a cell of $\mathcal{A}$. They initiated the study of the chromatic number of $\mathcal{H}_{\text {line-cell }}$, and proved that for $|L|=n, \chi\left(\mathcal{H}_{\text {line-cell }}\right)=O(\sqrt{n})$ and $\chi\left(\mathcal{H}_{\text {line-cell }}\right)=\Omega\left(\frac{\log n}{\log \log n}\right)$. In other words, they proved that the lines of every simple arrangement of $n$ lines can be colored with $O(\sqrt{n})$ colors so that there is no monochromatic face; furthermore, they provided an intricate construction of a simple arrangement of $n$ lines that requires $\Omega\left(\frac{\log n}{\log \log n}\right)$ colors.

In this short note, we improve their upper bound by a $\Theta(\sqrt{\log n})$ factor, and extend it to not necessarily simple arrangements.

Theorem 1. The lines of every arrangement of $n$ lines in the plane can be colored with $O(\sqrt{n / \log n})$ colors so that no face of the arrangement is monochromatic.

A set of points in the plane is in general position if it does not contain three collinear points. Let $\alpha(S)$ denote the maximum number of points in general position in a set $S$ of points in the plane, and let $\alpha_{4}(n)$ be the minimum of $\alpha(S)$ taken over all sets $S$ of $n$ points in the plane with no four point on a line. Erdös pointed out that $\alpha_{4}(n) \leqslant n / 3$ and suggested the problem of determining or estimating $\alpha_{4}(n)$. Füredi [3] proved that $\Omega(\sqrt{n \log n}) \leqslant \alpha_{4}(n) \leqslant o(n)$.

We observe that any improvement of the bound in Theorem 1 would immediately imply a better lower bound for $\alpha_{4}(n)$. Indeed, suppose that $\chi(A) \leqslant k(n)$ for any arrangement of $n$ lines, and let $P$ be a set of $n$ points, no four on a line. Let $P^{*}$ be the dual arrangement of a slightly perturbed $P$ (according to the usual point-line duality, see, e.g., $[2, \S 8.2]$ ). Color $P^{*}$ with $k(n)$ colors such that no face is monochromatic, let $S^{*} \subseteq P^{*}$ be the largest color class, and let $S$ be its dual point set. Observe that the size of $S$ is at least $n / k(n)$ and it does not contain three collinear points, since the three lines that correspond to any three collinear points in $P$ bound a face of size three in $P^{*}$.

\section{Proof of Theorem 1}

Let $\mathcal{A}$ be an arrangement of a set $L$ of $n$ lines, decomposing the plane into the set $C$ of cells, and let $\mathcal{H}_{\text {line-cell }}$ be the corresponding hypergraph (defined as in the previous section). We show that $\chi\left(\mathcal{H}_{\text {line-cell }}\right)=O\left(\sqrt{\frac{n}{\log n}}\right)$.

An independent set in $\mathcal{H}_{\text {line-cell }}$ is a set $S \subset L$ such that for every $c \in C, c$ is not a subset of $S$ (in other words, no cell of $\mathcal{A}$ has its boundary formed only by lines in $S$ ). The proof is based on the following fact. 
Theorem 2. There is an absolute constant $c>0$ such that the size $\alpha\left(\mathcal{H}_{\text {line-cell }}\right)$ of the maximum independent set is at least $c \sqrt{n \log n}$.

We color the lines in $\mathcal{A}$ so that no face is monochromatic by following the same method as in [1] (where they used the weaker version of Theorem 2 stating $\alpha\left(\mathcal{H}_{\text {line-cell }}\right)=\Omega(\sqrt{n})$ ). That is, we iteratively find a large independent set of lines (whose existence is guaranteed by Theorem 2), color them with the same (new) color, and remove them from $\mathcal{A}$.

Clearly, this algorithm produces a valid coloring. We verify, by induction on $n$, that at most $\frac{2}{c} \sqrt{n / \log n}$ colors are used in this coloring. We assume the bound is valid for all $n \leqslant 256$ (by taking sufficiently small $c>0$ ). For $n>256$, we have $\log 4<\frac{1}{4} \log n$. Let $i$ be the smallest integer such that after $i$ iterations the number of remaining lines is at most $n / 4$. Since in each of these iterations at least $c \sqrt{\frac{n}{4} \log \frac{n}{4}} \geqslant c \sqrt{\frac{n}{8} \log n}$ vertices (lines) are removed, $i \leqslant \frac{n / 4}{c \sqrt{\frac{n}{8} \log n}} \leqslant \frac{1}{\sqrt{2} c} \sqrt{n / \log n}$. Therefore, by the induction hypothesis the number of colors that the algorithm uses is at most

$$
\begin{aligned}
i+\frac{2}{c} \sqrt{\frac{\frac{n}{4}}{\log \frac{n}{4}}} & \leqslant \frac{1}{\sqrt{2} c} \sqrt{\frac{n}{\log n}}+\frac{1}{c} \sqrt{\frac{n}{\log n-\frac{1}{4} \log n}} \\
& <\frac{1}{\sqrt{2} c} \sqrt{\frac{n}{\log n}}+\frac{\sqrt{4 / 3}}{c} \sqrt{\frac{n}{\log n}}<\frac{2}{c} \sqrt{\frac{n}{\log n}} .
\end{aligned}
$$

The proof of Theorem 2 is based on a result on independent sets in sparse hypergraphs. Given a hypergraph $\mathcal{H}$ on a vertex set $V$, the sub-hypergraph $\mathcal{H}[X]$ induced by $X \subset V$ consists of all edges of $\mathcal{H}$ that are contained in $X$. A hypergraph $\mathcal{H}=(V, E)$ is $k$-uniform if every edge $e \in E$ has size $k$. Given a $k$-uniform hypergraph $\mathcal{H}$ and a set $S \subset V$ with $|S|=k-1$, the co-degree of $S$ is the number of all vertices $v \in V$ such that $S \cup\{v\} \in E$. Kostochka et al. [4] proved that if $\mathcal{H}$ is a $k$-uniform hypergraph, $k \geqslant 3$, with all co-degrees at most $d$, then $\alpha(\mathcal{H}) \geqslant c_{k}\left(\frac{n}{d} \log \frac{n}{d}\right)^{\frac{1}{k-1}}$, where $c_{k}>0$.

In fact, a careful look at their proof reveals the following result, that we state for 3-uniform hypergraphs, since this is the case that we need.

Lemma $2.1([4])$. Let $\mathcal{H}=(V, E)$ be a 3-uniform hypergraph on $|V|=n$ vertices with all co-degrees at most $d, d<n /(\log n)^{12}$. Let $X$ be a random subset of $V$, obtained by choosing each vertex of $V$ independently with probability $p=\frac{n^{-2 / 5}}{(d \log \log \log n)^{3 / 5}}$. Let $Z$ be a set chosen uniformly at random among all the independent sets of $\mathcal{H}[X]$. Then, with high probability $|Z|=\Omega(\sqrt{n \log n})$.

With Lemma 2.1 in hand we can now prove Theorem 2.

Proof of Theorem 2: A cell of an arrangement $\mathcal{A}$ is called an $r$-cell, if $r$ lines of $L$ are forming its boundary. Let $\mathcal{H}_{\triangle} \subset \mathcal{H}_{\text {line-cell }}$ be the 3-uniform hypergraph with the vertex set $L$ being the set of lines, and each hyperedge defined by the triple of lines forming the boundary of a 3 -cell of $\mathcal{A}$. Since any two lines can participate in the boundaries of at most four 3-cells of $\mathcal{A}$, all co-degrees of $\mathcal{H}$ are at most $d=4$. Now, as in Lemma 2.1, let $X$ be a random subset of $L$, obtained by choosing each line in $L$ independently with probability 
$p=\frac{n^{-2 / 5}}{(4 \log \log \log n)^{3 / 5}}$. Since there are $O\left(n^{2}\right)$ faces in $\mathcal{A}$ and $O(n)$ of them are 2-cells (since every line can bound at most four such faces), the expected number of 2-cells of $\mathcal{A}$ in $\mathcal{H}_{\text {line-cell }}[X]$ is $O\left(p^{2} n\right)=o(\sqrt{n \log n})$, and the expected number of $r$-cells, $r \geqslant 4$, of $\mathcal{A}$ in $\mathcal{H}_{\text {line-cell }}[X]$ is $O\left(p^{4} n^{2}\right)=o(\sqrt{n \log n})$. From Lemma 2.1 it follows that there exists a set $Z \subset X \subset L$ of size $\Omega(\sqrt{n \log n})$, that is an independent set of $\mathcal{H}_{\triangle}[X]$, and such that the number of $r$-cells, $r \neq 3$, of $\mathcal{A}$ in $\mathcal{H}_{\text {line-cell }}[Z]$ is $o(\sqrt{n \log n})$. Removing from $Z$ one vertex (line) for each such $r$-cell, we obtain an independent set of $\mathcal{H}_{\text {line-cell }}$ of size $\Omega(\sqrt{n \log n})$.

\section{References}

[1] P. Bose, J. Cardinal, S. Collette, F. Hurtado, S. Langerman, M. Korman, and P. Taslakian, Coloring and guarding arrangements, Discrete Mathematics and Theoretical Computer Science 15 (2013), 139-154. Also in: 28th European Workshop on Computational Geometry (EuroCG), March 19-21, 2012, Assisi, Perugia, Italy, 89-92.

[2] M. de Berg, O. Cheong, M. van Krefeld, M. Overmars, Computational Geometry: Algorithms and Applications, 3rd edition, Springer, 2008.

[3] Z. Füredi, Maximal independent subsets in Steiner systems and in planar sets, SIAM J. Disc. Math. 4 (1991), 196-199.

[4] A. Kostochka, D. Mubayi, J. Verstraëte, On independent sets in hypergraphs, Random Structures and Algorithms 44 (2014), 224-239. 